\title{
Review
}

\section{Analysis on Risk Factors of Nosocomial Infection in Orthopedic Patients and Research on Nursing Strategies}

Zhitao Guo

Department of Emergency Medicine, Tianjin Xiqing Hospital, Tianjin China

\author{
Keywords \\ orthopedic patients, trauma, nosocomial \\ infection \\ Correspondence \\ Zhitao Guo, \\ E-mail: guozhitaotj@sina.com \\ DOI: $10.1515 / i$ ii-2017-0125
}

\begin{abstract}
Orthopedic patients mostly comprise traumatic patients and elderly or sick individuals. More patients with emergency surgery suffer from open wounds and serious pollution, and operation time is relatively long. Thus, orthopedic patients with surgical incision infection account for a large proportion of incidence of hospital infection. Orthopedic patients are also bedridden for long periods, and they receive poor bone tissue blood supply. In surgical incision infections, mild cases suffer from delayed wound healing, whereas severe cases can form osteomyelitis. This study reviews progress of research on risk factors of nosocomial infection among orthopedic patients in recent years.
\end{abstract}

With rapid development of modern orthopedic medicine, an increasing trend was observed in wide use of broad-spectrum antibiotics and immunosuppressive agents, increase in elderly population and chronic diseases, development of invasive treatment technology, and infection rates in orthopedic hospitals. These conditions not only increase patient hospitalization time and costs but also endanger patient safety. This study reviews recent progress of research on risk factors of nosocomial infection of orthopedic patients.

\section{Risk factors of nosocomial infection}

\section{Risk factors and prevention of preoperative infection}

Some information indicated that prolonged hospital stay results in higher rate of wound infection; this condition is one of the combined risk factors for incision infection ${ }^{[1]}$. As a result, patients' preoperative hospital stay should be minimized as possible. Nutritional status of patients should be evaluated to improve and treat underlying diseases. Pretreatment and control can cause potential diseases of infection. Thus, improvement should focus on patients' immunity; such improvements may include correcting hypoxemia and hypoproteinemia. For patients with low immunity, supporting treatments should be considered.
Before surgery, skin of patients should be cleaned routinely, and infection should be avoided in curative patients. When cleaning the skin, damage to epidermis should be avoided. Literature reported that infection rates of razor wounds reaches $5.6 \%$, that of wounds caused by hair depilatory creams is $0.6 \%$, and that of postoperative infection of hairless parts totals $0.6 \%$. Another report indicated higher infection rate of preoperative shaving than morning shaving, implying their significant differences.

Antibiotics should be used to clean seriously infected wounds. Preventive medication should also be implemented in a strict and reasonable manner. Antibiotics should be used in accordance with cleanliness of the wound. Cleaning of wounds does not require preventive use of antibiotics. However, preventive medication must be applied to implantation of prosthesis, venerable age, malnutrition, and coexistence of multiple diseases. Cleanliness of contaminated wound requires usage of antibiotics. Nursing staff should be aware of diseases related to contaminated wounds. Preoperative pollution prevention necessitates use of antibiotics. A $30 \mathrm{~min}$ intravenous medication is the most appropriate before operations ${ }^{[2]}$.

Disinfection and monitoring of operating room are very important. The operating room should be cleaned between 
operations. Infection surgery should be performed in a regular operating room. Postoperative strict sterilization and disinfection and sterilization of surgical instruments and equipment should be performed in accordance with "disinfection technical specifications."

Medical personnel participating in operation should conduct preoperative preparations, including cutting of nails, changing of clothes, brushing of hands, and wearing of sterile gloves. Medical personnel with furuncle, eczema, upper respiratory tract infection, and dangerous bacteria (e.g., drug resistant Staphylococcus and Micrococcus scarlatinae) in nasopharynx and intestine should not enter the operating room before recovery ${ }^{[3]}$.

\section{Risk factors and prevention of infection during surgery}

Surgeons and nurses should correctly understand the use of relevant medical device and minimize operation time. When operation time reaches more than $4 \mathrm{~h}$, they should replace their gloves during operation and use antibiotics on patients. When patients need blood transfusion, autotransfusion is advocated rather than allogeneic transfusion as to prevent reduction of cell-mediated immunity. Electric surgery equipment should not be used as much as possible because electric knife cutting and coagulation hemostasis on tissues cause more significant damages than average scalpel. Surgery must be a strict aseptic operation. When gloves are punctured, they should be replaced with new ones immediately. For open bone surgery or transplant orthopedic surgery, medical personnel should wear two pairs of sterile gloves. When dealing with infected or contaminated parts, operators should replace their sterile gloves. Environmental health management of operating room should be strengthened. When surgery lasts for more than 3 $h$, circulating air disinfection machine should be opened for air disinfection. Medical workers (anesthesiologist and tour nurse) participating in operation cannot leave the operating room ${ }^{[4]}$.

\section{Risk factors and prevention of postoperative infection}

Dressing procedures should be strictly implemented. Wounds of patients with infectious wound or skin infection should not be redressed in ordinary dressing room. Patients should be quarantined appropriately. During treatment of wounds of different patients, medical workers should wash or disinfect their hands. When signs of infection exist, bacterial culture should be performed timely. Early pathogen monitoring should be performed for infectious wounds, and rational use of anti-infective drugs should be guided in accordance with drug susceptibility test results. Quarantine measures should be taken especially for infectious wounds. Medical staff suffering from infectious diseases, such as fever blister, group A Streptococcus, and Staphylococcus aureus infection, should not contact patient wounds. Management of ward sanitation should be strengthened, time should be ventilated, ward should be kept clean, and frequency of companionship should be reduced. Through monitoring of infected cases, the department of nosocomial infection management should provide feedback on surgical infection rate to doctors and department heads every month to improve doctors' awareness regarding infection ${ }^{[5]}$.

Policies on disinfection and isolation should be strictly carried out. Air disinfection in wards should be strictly monitored, and ventilation measures should be taken to improve air quality and reduce microbial content of air.

\section{Nursing strategies}

\section{Strengthening training on nosocomial infection and ward management}

Through training, medical staff may gain awareness of the importance of prevention of nosocomial infection. Rules and regulations should be strictly implemented. Patient management should be strengthened. Patients and their family members should be educated on hospital infection, and time of hospitalization should also be shortened. Frequency of companionship and visits should be restricted and reduced. Health education and guidance should be provided to patients and their families. Patients should be arranged in a quiet, clean, safe, and comfortable ward. Windows for ventilation should be regularly opened. Cleaning and disinfection must be performed, and room temperature of $18-22{ }^{\circ} \mathrm{C}$ should be maintained. Relative humidity should be $50 \%$ to $60 \%$. Visiting personnel or accompanying people with respiratory tract infection should be persuaded to suspend visits or advised to wear masks. 


\section{Strict disinfection}

Treatment rooms should be sterilized everyday for $1 \mathrm{~h}$, and wards should be ventilated twice every morning and evening to maintain freshness and dryness of indoor air. Ward grounds should be swept with 1:200 "84" disinfectant twice. A three-day and $0.2 \%$ oxyacetic acid solution should be used interchangeably. The bedside cabinet should also be scrubbed with a sterile towel once a day. When patients leave the hospital, care units must undergo strict terminal sterilization. Wet cleaning should be performed with a towel/table and quilt cover/bed, and effective dirt treatment system should be established. Disinfection and quarantine measures should be seriously implemented. Disinfection management on atomizer, oxygen humidification bottle, and oxygen catheter pipe should be strengthened to prevent pathogenic microorganisms. When signs of infection appear in wounds, bacterial culture should be performed right away. Pathogenic monitoring should also be conducted on infected wounds, and rational use of antiinfective drugs should be guided according to results of drug susceptibility test. Patients with especially infected wounds should be quarantined. Staff with infectious diseases, such as fever blister, group A Streptococcus, and Staphylococcus aureus infection, should not make contact with patient wounds. Management of ward sanitation should be strengthened, and wards must remain clean and ventilated. Frequency of companion should be reduced. Through monitoring of infected cases, the department of nosocomial infection management should provide doctors department heads with feedback on surgical infection rate every month to improve doctors' awareness of infection ${ }^{[5]}$. Policies on disinfection and isolation should be strictly carried out. Air disinfection in wards should be strictly monitored, and ventilation measures should be taken to improve air quality and reduce microbial content of air.

Aseptic operation should be strictly performed for deep vein catheterization and indwelling catheters. Wearing of sterile gloves and use of disposable suction tube when suctioning should be observed. Venous puncture indwelling needle should be replaced once a week to closely observe whether red, swollen, hot, pain symptoms manifest on punctured skin. When inflammation exists, bacterial culture of subcutaneous catheter should be performed as soon as possible. Medical staff should thoroughly wash or disinfect their hands before and after treatment ${ }^{[6]}$.

\section{Preventing unnecessary invasive operations}

Invasive operation can damage defensive barrier of the body and increase chances of nosocomial infection to critically ill patients with low immunity. Thus, we should avoid unnecessary invasive operation to reduce incidence of nosocomial infection. With permission of patients, all indwelling pipelines should be removed immediately to shorten the time of intubation and reduce nosocomial infection.

\section{Shortening time of hospitalization and using antibiotics in a reasonable manner}

Universal education and knowledge update of clinicians' rational application of antibiotics should be strengthened. Antibiotics should be strictly used, controlled, and monitored. Sensitive antibiotics should be selected according to drug sensitivity test and timely withdrawal to prevent double infection and drug resistance. Patients should be provided with psychological counseling and informed of increased possibility of cross infection with long-term hospitalization. Patients should leave the hospital after recovery from their illnesses. In hospitalization of elderly people, primary diseases should be treated and given attention to improve immunity of elderly patients, strengthen support therapies, and improve epidemic prevention function of the body.

\section{Declarations}

\section{Acknowledgements}

No.

\section{Competing interests}

The author declares that he has no competing interest.

\section{Authors' contributions}

ZT Guo made the literature analysis and wrote, discussed and revised the manuscript of this review.

\section{References}

1 Hao M. Prevention and Control of Neurological Nosocomial Infection of 
Elderly Patients. Chemists, 2011, 6: 173.

Gao R, Liu Y, Zhao G, et al. Investigation and Analysis of Nosocomia Infection in Stroke Patients. Journal of Nursing Administration, 2013, 3: $17-18$.

3 Peng D, Huang $\mathrm{H}, \mathrm{Wu}$ J, etc. Characteristics of Acquired Lung Infection in Stroke Patients. Chinese Journal of Hospital Infection, 2012, 8: 591-593.

4 Zhang L. Clinical Analysis and Nursing Countermeasures of Nosocomial
Infection of Elderly Intensive Care Unit, Department of Neurology. Nursing Practice and Research, 2011,6: 35-36.

5 Guo Y, Chen W, Zhang Z, et al. Investigation and Analysis of Risk Factors of Incision Infection of Aseptic Operation. Chinese Journal of Nosocomiology, 2011,4: 394 - 396.

6 Feng X, Guo X, Zhai R. Analysis on 3-year Nosocomial Infection of Shanxi Provincial Cancer Hospital. Chinese Journal of Nosocomiology, 2012, 8: 585-5586. 